\title{
An intertwining of a hereditary algebra and a cohereditary coalgebra
}

\author{
Hanni Garminia*, Pudji Astuti and Irawati \\ Faculty of Mathematics and Natural Sciences, Institut Teknologi Bandung, Jln. Ganesha 10 Bandung 40132, Indonesia \\ *To whom correspondence should be addressed. E-mail: hani@math.itb.ac.id
}

Received 18 October 2007

http://dx.doi.org/10.11113/mjfas.v4n1.35

\section{ABSTRACT}

Let $F$ be a field. It is known that if $A$ is a finitely dimensional hereditary $F$-algebra then the dual $A^{*}=H_{F}(A, F)$ is a cohereditary $F$-coalgebra. On the other hand if $C$ is a finitely dimensional cohereditary $F$-coalgebra then the dual $C^{*}=\operatorname{Hom}_{F}(C, F)$ is a hereditary $F$-algebra. As a result, if $C$ is a finitely dimensional $F$-coalgebra then $C$ is a cohereditary $F$-coalgebra if and only if the dual $C^{*}$ is a hereditary $F$-algebra. In this paper we enlarge the class of algebras and coalgebras under consideration. Particularly we show that properties similar to the above results are obtained for a class of algebras and coalgebras over a self injective commutative ring $R$ where the algebras and coalgebras, as $R$-modules, are locally projective.

| Algebra | Coalgebra | Cohereditary | Hereditary |

\section{Introduction}

This article explains an intertwining of a hereditary algebra and a cohereditary coalgebra through their duals dan the global dimension of the underlying ring. The notion of a cohereditary coalgebra is adopted from [9] that some articles call a hereditary coalgebra (see [2], [4], [7]). The fact that there is a faithful functor from the comodule category to a full subcategory of a module category [1] will be exploited to derive the result.

Some results have been discussed regarding cohereditary coalgebras. For example Natasescu, Torrecillas, and Zhang [7] investigated properties of cohereditary coalgebras including their duals and the direct sum of these coalgebras. Jara, Merino, Liena, and Stefan [4] discussed a relationship between cohereditary coalgebras and formally smooth coalgebras. Further, Chin [2] showed that a pointed cohereditary coalgebra and the path algebra of its quiver are isomorphic. However, all of these works deal with coalgebras over a field.

In case of the underlying ring is a field, we have that the dual of a finitely dimensional hereditary algebra is a cohereditary coalgebra. That is, let $A$ be an algebra over a field $F$. If $A$ is a finitely dimensional hereditary $F$-algebra then its dual $A^{*}=\operatorname{Hom}_{F}(A, F)$ is a cohereditary $F$-coalgebra. On the other hand if $C$ is a 
finitely dimensional cohereditary $F$-coalgebra then its dual $C^{*}=\operatorname{Hom}_{F}(C, F)$ is a hereditary $F$-algebra. As a result, if $C$ is a finitely dimensional $F$-coalgebra then $C$ is a cohereditary $F$-coalgebra if and only if its dual $C^{*}$ is a hereditary $F$-algebra [7].

In this paper we enlarge the class of algebras and coalgebras under consideration. Particularly we investigate properties similar to the above results for a class of algebras and coalgebras over a self injective commutative ring $R$ where as $R$-modules they are locally projective. A field is a self injective ring but the converse does not hold. In this larger class we shall show that the dual of a finitely generated hereditary $R$-algebra is a cohereditary coalgebra. Conversely, let an $R$-coalgebra $C$, as an $R$-module be locally projective. Then $C$ is a cohereditary coalgebra if and only if its dual $C^{*}$ is a hereditary algebra.

To begin with, notation $R$ stands for a commutative ring with a unit and $F$ stands for a field. The notations ${ }_{R} \mathbf{M}$ and $\mathbf{M}^{C}$ denote respectively the category of (left) $R$-modules and the category of right comodules over a coalgebra $C$. The full subcategory of ${ }_{R} \mathbf{M}$ consisting of all $M$-subgenerated $R$-modules, for some $M$ an object in ${ }_{R} \mathbf{M}$, is denoted by $\sigma[M]$.

An $R$-coalgebra $C$ is called cohereditary if it is a cohereditary $C$-comodule. The notion of a cohereditery $C$-comodule is defined through injective comodules. Akin to an injective object in category theory, an injective comodule is defined as the following. Let $\operatorname{Hom}^{C}(M, N)$ denote the abelian group consisting of all $C$ comodule morphisms from $M$ to $N$.

Definition 1. Let $Q$ be in $\mathbf{M}^{C}$.

1. The $C$-comodule $Q$ is said to be $B$-injective in $\mathbf{M}^{C}$ for some $B$ in $\mathbf{M}^{C}$ if for every monomorphism $f: M \rightarrow B$, the canonical map $\operatorname{Hom}^{C}(f, A): \operatorname{Hom}^{C}(B, A) \rightarrow \operatorname{Hom}^{C}(M, A)$, where $\operatorname{Hom}^{C}(f, A)(\alpha)=\alpha \circ f$ for all $\alpha$ in $\operatorname{Hom}^{C}(B, A)$, is surjective .

2. The $C$-comodule $Q$ is said to be injective if $Q$ is $B$-injective for all $B$ in $\mathbf{M}^{C}$.

3. The $C$-comodule $Q$ is called cohereditary if every factor $C$-comodule of $Q$ is injective in $\mathbf{M}^{C}$.

An $F$-coalgebra $C$ is an example of an injective $C$-comodule [3]. If the field $F$ is substituted by a commutative ring $R, C$ has not to be injective. An $R$-coalgebra $C$ is an injective $C$-comodule provided $C$ is a flat $R$-module and $R$ is a self injective ring [1].

The following results, which can be found in [1], compare two categories $\mathbf{M}^{C}$ and ${ }_{C^{*}} \mathbf{M}$ where $C^{*}$ is the dual ring consisting of all $R$-module morphisms from $C$ to $R$. The results show that there is a faithful functor from $\mathbf{M}^{C}$ to ${ }_{C^{*}} \mathbf{M}$. Particularly, $\mathbf{M}^{C} \cong \sigma\left[{ }_{C^{*}} C\right]$ if and only if ${ }_{R} C$ is locally projective.

Theorem 2. [1] The following statements are equivalent

1. $\mathbf{M}^{C} \cong \sigma\left[{ }_{C^{*}} C\right]$

2. $\mathbf{M}^{C}$ is isomorphic by a functor with a full subcategory of ${ }_{C^{*}} \mathbf{M}$.

3. For every $M$ and $N$ in $\mathbf{M}^{C}, \operatorname{Hom}^{C}(M, N) \cong_{C^{*}} \operatorname{Hom}(M, N)$ 
4. ${ }_{R} C$ is locally projective.

\section{Global Dimension}

It was mentioned before that our goal is investigating the relationship between a hereditary algebra and a cohereditary coalgebra by using properties of the global dimension of the ring. In this section we review some results about this notion. This notion is introduced through projective dimensions of modules. We are also able to define global dimension from injective dimensions of modules. For that reason we shall study properties of projective and injective dimensions.

Let us divide ${ }_{R} \mathbf{M}$ into equivalent classes with the following relation. Let $A$ and $A^{\prime}$ be in ${ }_{R} \mathbf{M}$. We denote $A \square A^{\prime}$ if there are projective $R$-modules $P$ and $P^{\prime}$ so that $A \oplus P \cong A^{\prime} \oplus P^{\prime}$. It can be shown that the relation $\square$ is an equivalent relation on ${ }_{R} \mathbf{M}$. Hence we obtain the collection of equivalent classes $\mathbf{A}=\left\{[A] \mid A \in_{R} \mathbf{M}\right\}$ with $[A]=\{B \mid B \square A\}$.

Further, we can define a mapping on the above collection of equivalent classes. Consider that if $P$ is a projective $R$-module then $P \square 0$. We also know that if $A$ is in ${ }_{R} \mathbf{M}$, then there is an exact sequence

$$
0 \rightarrow B \rightarrow P \rightarrow A \rightarrow 0
$$

for some projective $R$-module $P$. Hence we can define a mapping $\mathbf{K}: \mathbf{A} \rightarrow \mathbf{A}, \mathbf{K}([A])=[B]$ where $[A],[B] \in \mathbf{A}, P$ is projective and satisfies (1).

In the following, we show that the mapping $\mathbf{K}: \mathbf{A} \rightarrow \mathbf{A}$ is well defined. Let $[A]=\left[A^{\prime}\right]$ be in $\mathbf{A}$ and consider the following exact sequences

$$
0 \rightarrow B \rightarrow P \rightarrow A \rightarrow 0 \text { and } 0 \rightarrow B^{\prime} \rightarrow P^{\prime} \rightarrow A^{\prime} \rightarrow 0
$$

where $P$ and $P^{\prime}$ are projective R-modules. Since $A \square A^{\prime}$, there are projective $R$-modules $Q, Q^{\prime}$ so that $A \oplus Q \cong A^{\prime} \oplus Q^{\prime}$. Let us consider the following exact sequences

$$
0 \rightarrow B \oplus Q \rightarrow P \oplus Q \rightarrow A \oplus Q \rightarrow 0 \quad \text { and } \quad 0 \rightarrow B^{\prime} \oplus Q^{\prime} \rightarrow P^{\prime} \oplus Q^{\prime} \rightarrow A^{\prime} \oplus Q^{\prime} \rightarrow 0 .
$$

Since $\quad A \oplus Q \cong A^{\prime} \oplus Q^{\prime} \quad$ then $\quad(B \oplus Q) \oplus\left(P^{\prime} \oplus Q^{\prime}\right) \cong\left(B^{\prime} \oplus Q^{\prime}\right) \oplus(P \oplus Q)$. In other word $B \oplus\left(Q \oplus P^{\prime} \oplus Q^{\prime}\right) \cong B^{\prime} \oplus\left(Q^{\prime} \oplus P \oplus Q\right)$. It means $B \square B^{\prime}$.

The above mapping provide us a mean for defining the global dimension of a ring.

\section{Definition 3.}

1. Let $A$ be in ${ }_{R} \mathbf{M}$. The projective dimension of $A$, written by $\operatorname{proj.dim}(A)$, is the smallest non negative integer $n$ such that $\mathbf{K}^{n}([A])=0$. Otherwise proj.dim $(A)$ is infinite.

2. The left global dimension of $R$, written by $\operatorname{l.glob} \cdot \operatorname{dim}(R)$, is the supremum of $\operatorname{proj} \cdot \operatorname{dim}(A)$ for all 
$A$ in ${ }_{R} \mathbf{M}$.

The global dimension notion can be used to characterize the hereditary property of a ring as shown in the following lemma.

Lemma 4. The ring $R$ is hereditary if and only if $\lg \operatorname{glob} \cdot \operatorname{dim}(R) \leq 1$.

\section{Proof}

$(\Rightarrow)$ Let $M$ be an $R$-module. Since every $R$-module is a homomorphic image of a free $R$-module, suppose $M$ is a homomorphic image of a free $R$-module $P$. Since every free $R$-module is projective, we have the following exact sequence,

$$
0 \rightarrow B \rightarrow P \rightarrow M \rightarrow 0
$$

The assumption that $R$ is left hereditary ring and $P$ is a projective $R$-module imply that $P$ is hereditary. Hence $B$ is also a projective $R$-module. Hence $\operatorname{proj} \operatorname{dim}(B)=0$. Then we obtain that $[0]=\mathbf{K}^{0}([B])=[B]=\mathbf{K}([M])$. This implies that $\operatorname{proj} \cdot \operatorname{dim}(M) \leq 1$ for every $R$-module $M$. In other word $\operatorname{l.gl} \operatorname{dim}(R) \leq 1$.

$(\Leftarrow)$ Let $I$ be a left ideal of $R$. Let us consider the following exact sequence

$$
0 \rightarrow I \rightarrow R \rightarrow R / I \rightarrow 0
$$

It is clear that $R$ is a projective $R$-module and $\operatorname{proj} \cdot \operatorname{dim}(R / I) \leq \lg \operatorname{gl} \cdot \operatorname{dim}(R) \leq 1$.

In case the $\operatorname{proj} \cdot \operatorname{dim}(R / I)=0$ we have $\operatorname{proj} \cdot \operatorname{dim}(I)=0$.

In case $\operatorname{proj} \cdot \operatorname{dim}(R / I)=1$ we have $\operatorname{proj} \cdot \operatorname{dim}(R / I)=1-1=0$.

Hence $I$ is a projective $R$-module. We conclude $R$ is a hereditary ring.

In the following we will see that the global dimension of a ring can also be defined through injective dimensions of modules. We know that the injective property is the dual of the projective property. Hence the injective dimension can also be considered as the dual of the projective dimension.

Let $A$ and $A^{\prime}$ be in ${ }_{R} \mathbf{M}$. We write $A \square A^{\prime}$ if and only if there are injective $R$-modules $Q$ and $Q^{\prime}$ so that $A \oplus Q \cong A^{\prime} \oplus Q^{\prime}$. It is clear that the relation $\square$ is an equivalent relation. Hence we obtain the collection of equivalent classes $\mathbf{B}=\left\{[A] \mid A \in_{R} \mathbf{M}\right\}$ with $[A]=\{B \mid B \square A\}$. Note that if $Q$ is a injective $R$-module then $Q \square 0$. We also know that if $A$ is in ${ }_{R} \mathbf{M}$, then there is an exact sequence $0 \rightarrow A \rightarrow P \rightarrow B \rightarrow 0$ with $Q$ is injective. Hence we can define a mapping $\mathbf{S}: \mathbf{B} \rightarrow \mathbf{B}$ by $[A] \mapsto[B]$.

Definition 5. Let $A$ be in ${ }_{R} \mathbf{M}$. The injective dimension of $A$, written by $\operatorname{inj} \cdot \operatorname{dim}(A)$, is the smallest non negative integer $n$ such that $\mathbf{S}^{n}([A])=0$. 
A connection between injective dimension and global dimension notions is shown in the following lemma.

Lemma 6. Let $k$ is the supremum of $\operatorname{inj} \cdot \operatorname{dim}(A)$ for every $A$ in ${ }_{R} \mathbf{M}$, then $k$ is $\lg \operatorname{glob.dim}(R)$.

The previous lemma says that we can define the global dimension of a ring through injective dimensions of all modules over the ring.

Now we shall introduce global dimension notion of a $R$-coalgebra $C$ through injective dimensions of $C$ comodules. Let $C$, as $R$-module, be locally projective. It follows by Theorem 2 that the $C$-comodule category is isomorphic by a functor to a full subcategory of $C^{*}$-module, that is $\sigma\left[{ }_{C^{*}} C\right]$. It means every $C$-comodule is a $C^{*}$-module in $\sigma\left[{ }_{C^{*}} C\right]$ and every $C^{*}$-module in $\sigma\left[{ }_{C^{*}} C\right]$ is a $C$-comodule. Hence we can define the injective dimension of a $C$-comodule and the left global dimension of $C$.

Definition 7. Let the $R$-coalgebra $C$, as $R$-module, be locally projective .

1. The injective dimension of $A$, denoted by inj.dim ${ }^{C}(A)$, is defined to be inj.dim $C^{*}(A)$, for every $A$ in $\mathbf{M}^{C}$.

2. The right global dimension of $C$, written by r.glob.dim(C), is defined as the supremum of inj.dim ${ }^{C}(A)$ for all $A$ in $\mathbf{M}^{C}$.

Lemma 8. Let $\operatorname{inj} \cdot \operatorname{dim}(R)=0$ and $C$ be a $R$-coalgebra which, as $R$-module, is locally projective. Then $C$ is a cohereditary $R$-coalgebra if and only if $r \cdot \operatorname{glob} \cdot \operatorname{dim}(C) \leq 1$.

\section{Proof}

$(\Rightarrow)$ Let $M$ be a $C$-comodule. It is clear that $M$ is a $C^{*}$-module in $\sigma\left[{ }_{C^{*}} C\right]$. It follows by [8] that every $C^{*}$-module in $\sigma\left[{ }_{C^{*}} C\right]$ is embedded in an injective $C^{*}$-module in $\sigma\left[{ }_{C^{*}} C\right]$. Let $M$ be embedded in an injective $C^{*}$-module $Q$. Let us consider the following exact sequence in $\mathbf{M}^{C}$

$$
0 \rightarrow M \rightarrow Q \rightarrow Q / M \rightarrow 0
$$

Since $C$ is a locally projective $R$-module, then $\mathbf{M}^{C} \cong \sigma\left[{ }_{C^{*}} C\right]$. Remember that $C$ is a left cohereditary coalgebra. In addition, we already have that $Q$, as $C$-comodule, is injective. Hence, referring to [6] we obtain $Q$ is a cohereditary $C$-comodule. Furthermore $Q / M$ is an injective $C$-comodule. According to [6], $Q / M$ is an injective $C^{*}$-module. Hence $\operatorname{inj} \operatorname{dim}(Q / M)=0$. Then we obtain that $[0]=\mathbf{S}^{0}([Q / M])=[Q / M]=\mathbf{S}([M])$. This implies that $\operatorname{inj} \cdot \operatorname{dim}(M) \leq 1$ for every $C^{*}$-module $M$. In other word $\operatorname{l} \cdot g l \cdot \operatorname{dim}\left(C^{*}\right) \leq 1$. Thus we conclude that $r \cdot \operatorname{gl} \cdot \operatorname{dim}(C) \leq 1$

$(\Leftarrow)$ Let $I$ be a left coideal of $C$. It is clear that $I$ is a $C$-comodule and the sequence

$$
0 \rightarrow I \rightarrow C \rightarrow C / I \rightarrow 0
$$


is exact in $\mathbf{M}^{C}$. Since $\operatorname{inj} \cdot \operatorname{dim}(R)=0$, the $R$-coalgebra $C$ is an injective $C$-comodule. It is clear that $\operatorname{inj} \cdot \operatorname{dim}(I) \leq r . g l \cdot \operatorname{dim}(C) \leq 1$. In case $i n j \cdot \operatorname{dim}(I)=0$ we have inj.dim( $(C / I)=0$. In case $\operatorname{inj} \cdot \operatorname{dim}(I)=1$ we have inj.dim$(C / I)=1-1=0$. Hence $C / I$ is an injective $C$-comodule. We conclude $C$ is a cohereditary $R$-coalgebra.

\section{Hereditary Algebra and Cohereditary Coalgebra}

Consider that for the case the underlying ring is a field $F$, a finitely dimensional $F$-coalgebra $C$ is cohereditary if and only if the dual $C^{*}=\operatorname{Hom}_{F}(C, F)$ is a hereditary $F$-algebra [7]. Further, we observe that the dual of this result also holds for an $F$-algebra. That is, a finitely dimensional $F$-algebra $A$ is hereditary if and only if $A^{*}=\operatorname{Hom}_{F}(A, F)$ is a finitely dimensional cohereditary coalgebra. This is true since every an $F$ coalgebra is an injective comodule over itself. This observation inspire us to investigate these intertwining properties for a larger class of algebras and coalgebras.

In the following we show that the above results hold for algebras and coalgebras over a self injective commutative ring $R$ where, as $R$-modules, they are locally projective. A field is a self injective commutative ring and a finitely dimensional vector space is a locally projective module. From now on we assume $R$ is self injective.

A relation between $R$-algebra ( $R$-coalgebra) and its dual has been discussed in Brzezinski and Wisbauer [1]. If $C$ is an $R$-coalgebra then its dual $C^{*}$ will hold $R$-algebra structure. In contrast, the dual of any $R$ algebra does not form $R$-coalgebra. Nevertheless, the dual of an $R$-algebra $A$, denoted by $A^{*}$, is an $R$ coalgebra provided $A$ is finitely generated and projective. Hence, we restrict our result to finitely generated pojective $R$-algebras.

Theorem 9. Let $A$ be a finitely generated projective $R$-algebra. The dual $R$-coalagebra $A^{*}$ is cohereditary if and only if the $R$-algebra $A$ is hereditary.

\section{Proof}

According to Lemma 8 we obtain $\lg \operatorname{gl} \operatorname{dim}(A) \leq 1$. Further, $A$ being a finitely generated left hereditary $R$ algebra results in $C=A^{*}$ being a finitely generated projective $R$-module. Hence $\mathbf{M}^{C} \square_{C^{*}} \mathbf{M}$ and r.gl.dim $(C)=l . g l \cdot \operatorname{dim}\left(C^{*}\right)=1 . g l \cdot \operatorname{dim}(A) \leq 1$. Thus, according to Lemma 8 , this last inequality implies that $C=A^{*}$ is a cohereditary $R$-coalgebra.

The preceding theorem provides means to construct examples of cohereditary coalgebras over a self injective commutative ring. For example the ring $\square_{n}$ is self injective and the ring $A=\left(\begin{array}{cc}\square_{n} & \square_{n} \\ 0 & \square_{n}\end{array}\right)$ is a hereditary $\square_{n}$ algebra.

Hence $A^{*}=\operatorname{Hom}\left(A, \mathbf{Z}_{n}\right) \cong M_{(3 \times 1)}\left(\mathbf{Z}_{n}\right)=\mathbf{Z}_{n}^{3}$ is a cohereditary $\square_{n}$-coalgebra. 
Unlike the dual coalgebra, we always be able to construct the dual algebra from any coalgebra [1]. Hence, we can delete the finiteness condition in our result for the dual algebra as in the following.

Theorem 10. Let $C$ be an $R$-coalgebra where as $R$-module $C$ is locally projective. The dual $R$-algebra $C^{*}$ is hereditary if and only if the $R$-coalgebra $C$ is cohereditary.

\section{Proof}

According to Lemma 8 we obtain r.glob.dim $(C) \leq 1$. Hence r.glob.dim $\left(C^{*}\right) \leq 1$. Consequently, referring to Lemma $8, C^{*}$ is a hereditary ring. Since $R$ is embedded in the hereditary ring $C^{*}$, we have $C^{*}$ is a hereditary $R$-algebra.

Note that condition $R$ being self injective in part of Theorem 10, particularly the sufficient conditions for the dual hereditary $R$-algebra, actually can be omitted.

\section{Concluding Remarks}

We have investigated that an interwining of hereditary and cohereditary properties, which holds for Algebras and coalgebras over a field, also works for a larger class of Algebras and coalgebras over a self injective and commutative ring. Connecting hereditary and cohereditery properties with other properties such as strongly prime and dedekind domain is also an interesting topics which currently under inverstigation.

\section{Acknowledgement}

This research supported by ITB according to Surat Perjanjian Pelaksanaan Penelitian No.:174/K01.07/PL/07, 10 Januari 2007.

\section{References}

[1] Brzezinski T and Wisbauer R, Corings and comodules. London Mathematical Society Lecture Note Series, 309. Cambridge University Press, Cambridge, (2003)

[2] Chin W. "Hereditary and Path Coalgebras", Comm. algebra 30,(2002) 1829-1831

[3] Dascalescu S, Nastasescu C and Raianu S, Hopf Algebras. An Introduction. Monographs and Textbooks in Pure and Applied Mathematics, 235. Marcel Dekker, Inc., New York, (2001)

[4] Jara L, Merino L, Llena D, and Stefan D, "Hereditary and Formally Smooth Coalgebras", Algebras and Representation Theory, 8(3) (2005) 363-374

[5] Garminia $\mathrm{H}$ and Astuti P, "Karakterisasi Modul $\sigma[M]$-Koherediter", Majalah Ilmiah Himpunan Matematika Indonesia, (2006), 12(2), 225-231.

[6] Garminia H, Astuti P, and Irawati "Properties of Cohereditary Comodules", Journal of Mathematics and Science, (2007), 12(2).

[7] Natasescu C, Torrecillas B and Zhang Y H, "Hereditary coalgebras". Comm. Algebra 24 (1996) 1521-1528

[8] Wisbauer R, Foundations of module and ring theory. A handbook for study and research. Gordon and Breach SciencePublishers, Philadelphia, PA, (1991)

[9] Wisbauer R, "Tilting in module categories". Lecture Notes in Pure and Appl. Math., 201, Dekker, New York, (1998) 421-444. 